\title{
PSICOLOGÍA
}

\section{Caracterización de pacientes internados con trastornos de personalidad}

\author{
Marcos Hernán Capurro1, Andrés Arce², Cristina Arrom², \\ Carmen Marina Arrom Suhurt ${ }^{3}$, María Auxiliadora Arrom³, \\ Jessica Rivera², Christian Hermida ${ }^{2}$
}

\section{Resumen}

Introducción: El término personalidad hace referencia a las características estables de un individuo y que se muestran en su forma de comportarse. Los trastornos de la personalidad (TP) son desviaciones extremas de la personalidad y han cobrado relevancia por su alta incidencia y por su presentación con otros trastornos mentales. La personalidad ha sido definida de forma general como la totalidad de los rasgos emocionales y conductuales que caracterizan a una persona en condiciones normales. Los TP son variantes de los rasgos de personalidad, inflexibles y desadaptativos, que causan o bien un deterioro funcional significativo o un malestar subjetivo. Se presenta una forma estable de comportamiento caracterizado por maneras de percibir e interpretar la realidad, afectividad y emociones, relaciones interpersonales y manejo de los impulsos apartados de lo que se espera culturalmente de la persona. La personalidad y sus trastornos resultan de la interacción de factores genéticos con otros determinados por la educación y crianza. La experiencia clínica señala que características anormales de la personalidad tienden a normalizarse a medida que el sujeto alcanza la edad media de la vida. Pero hay otros casos en los que en la senectud, los rasgos anormales de la personalidad pueden agravarse de nuevo y ser fuente de problemas para pacientes u cuidadores. Todos los trastornos de la personalidad han sido diagnosticados con cifras similares en todas las culturas del mundo. La prevalencia estimada de TP en la comunidad varía ampliamente según distintos estudios, desde un 5,9 hasta un $22,5 \%$. No obstante, los TP son 4 veces más prevalentes en muestras clínicas que entre la población general. Representa el $10 \%$ de los pacientes ambulatorios y el $20 \%$ de los pacientes hospitalizados alcanzando el $49 \%$ en adolescentes internados. Las personas con patologías severas de la personalidad hacen un mayor uso de los servicios de salud mental.

Objetivo: Caracterizar a pacientes internados y reingresados con trastornos de

\footnotetext{
1. Centro Nacional de Control de Adicciones, Ministerio de Salud Pública y Bienestar Social, Paraguay.

2. Cátedra de Psiquiatría de la Facultad de Ciencias Médicas, UNA, Paraguay.

3. Centro para el Desarrollo de la Investigación Científica, CEDIC, Paraguay.

E-mail: marcoshcapurro@hotmail.com

DOI: $10.26885 /$ rcei.foro.2017.115
} 
personalidad en un Servicio ambulatorio de Psiquiatría.

Metodología: Estudio descriptivo, retrospectivo, de fuente secundaria. Se utilizó registro de internados del Servicio de la Cátedra de Psiquiatría (2004 a 2009) y sus fichas. Se procesó información con SPSS. Para diagnóstico, la Clasificación internacional de enfermedades, décima versión CIE-10.

Resultados: Sobre un total de 426 pacientes, 99 de los mismos tenían trastornos de personalidad, el $78 \%$ (77) fue de sexo femenino y $22 \%$ (22) masculino. La relación varón/mujer entre pacientes internados sin TP (327) fue menos acentuada, 61\% (200) fueron de sexo femenino y 39\% (127) masculino. El $63.6 \%$ (63) tenía hasta 30 años de edad y el grupo restante $36.4 \%$ (36) 31 y más años. Los principales motivos de internación de los pacientes fueron: $35.3 \%$ (35) riesgo suicida, $31.3 \%$ (31) tentativa suicida, $11.1 \%$ (11) poco control de los impulsos, nerviosismo, conducta agresiva e irritabilidad, agitación psicomotriz $4 \%$ (4) y $18.1 \%$ (18). Un $24.2 \%$ (24) del total de pacientes con TP tuvo un primer reingreso por riesgo suicida en $62.5 \%$ (15) y por tentativa suicida $12.5 \%$ (3) principalmente. Sólo 6\% (6) tuvo un segundo reingreso y $2 \%$ (2) un tercer y cuarto reingreso por riesgo suicida.

Conclusiones: se evidenció una acentuada tendencia de género femenino entre pacientes con TP en relación a aquellos internados sin TP, casi duplicando dicha relación (3,5:1 versus 1,8:1). El hallazgo de una relación mayor de los trastornos de la personalidad con el sexo femenino en comparación con el masculino coincide con la información encontrada en otros estudios de la región. De la misma manera se observó un mayor número de internaciones en el rango de edad comprendido entre los 20 y 30 años, dato que va de la mano con las cifras recogidas en trabajos similares, demostrando que la gravedad de los trastornos de la personalidad tiende a disminuir con la edad y con ello la necesidad de medidas como la internación. Los principales motivos de internación y re internación fueron el riesgo suicida, la tentativa suicida y la falta de control de impulsos con conducta agresiva. Asimismo como en se puede encontrar en otras referencias, para la mayoría de los pacientes fue su primera internación y alrededor de un cuarto de los pacientes requirieron ser readmitidos. Tanto en la primera como en las sucesivas internaciones los pacientes presentaban algún nivel de riesgo suicida (por arriba del 60\%) en resonancia con otros estudios.

Palabras clave: trastorno de personalidad, género, internación, re internación.

\section{REFERENCIAS}

Kaplan, S. (2014). Sinopsis de Psiquiatría (11a ed.). New York: Lippincott Williams and Wilkins.

Leiderman, E., Buchovsky, S., Jiménez, M., Nemirovsky, M., Pavlovsky, F., Giordano, S. \& Lipovetzky, G. (2004). Diagnóstico y tratamiento del trastorno Borderline de la personalidad: una encuesta a profesionales. VERTEX, 15(58), p. 280-286. 
Caracterización de pacientes internados con trastornos de personalidad. Capurro et al.

Organización Mundial de la Salud. (2001). La salud pública al servicio de la salud mental. Recuperado de http://www.who.int/whr/2001/en/ whr01_ch1_es.pdf

Organización Mundial de la Salud. (2013). Plan de Acción sobre Salud Mental 20132020. Ginebra. Recuperado de http://www.who.int/topics/mental_health/es/ index.html

Szerman Bolotner, N., Peris Díaz, L. (2008). Cannabis y trastornos de personalidad (pp. 89-103). En: F Arias Horcajadas, J. A. Ramos Atance (Ed.), Aspectos psiquiátricos del consumo de cannabis: casos clínicos. Madrid: Sociedad Española de Investigación en Cannabinoides, CEMA.

Verheul, R. (2001). Comorbidity of personality disorders in individuals with substance use disorders. Eur Psychiatry, 16, 274-82. 